neurological and psychiatric manifestations in 20.2\% and $6.7 \%$ respectively. Associated auto immune diseases were Sjogren syndrome in $14.6 \%$, rheumatoid arthritis in $6.7 \%$ and thyroiditis in $7.8 \%$. Biological findings showed leucopenia in $48.8 \%$, thrombopenia in $26.2 \%$ and hemolytic auto immune anaemia in $3.6 \%$. Immunological screening revealed positive anti nuclear antibodies in 92.1\%, anti DNA antibodies in $84.3 \%$, anti $\mathrm{Sm}$, anti nucleosome and anti phospholipid antibodies in $40.8 \%, 31.6 \%$ and $36.6 \%$ respectively. SLE activity was assessed by SLEDAI score which mean value was 9.1. Relapses occurred in $39.3 \%$ of the patients and remission was recorded in 56\%. Four patients died. Infection occurred in $18.8 \%$ of the cases, steroid induced diabetes in $12.9 \%$ and osteoporosis in $16.5 \%$.

Conclusions In our series, SLE patients had a high prevalence of cutaneous and articular manifestations. Nephritis lupus prevalence was similar to other African, Afro-american and Hispanic groups and lower than Asians. Global outcome was good with more than a half remission.

\section{PS2:36 PREVALENCE OF THYROID DISEASES IN SLE PATIENTS AMONG SAUDI POPULATION}

I Al-Homood, R AlKhathami, M AlYousef, S AlJohani. King Fahad Medical City. Medical Specialities Department. Rheumatology section, Riyadh, Saudi Arabia

\subsection{6/lupus-2018-abstract.84}

Background Systemic Lupus Erythematosus (SLE) is an autoimmune disease that can affect different systems. Thyroid dysfunction is common in SLE. Several studies reported a varied prevalence of thyroid diseases in SLE patients.

Objective To report the prevalence of thyroid dysfunction in Saudi patients with SLE.

Methods Retrospective review of medical charts of SLE patients who attended rheumatology clinic at King Fahad Medical City between 2014 and 2015 was conducted.The primary outcome was the prevalence of thyroid dysfunction; the secondary outcome was the association between SLE and thyroid diseases. Pearson's chi-squared test was used to compare the distribution of thyroid diseases, and a student t-test was used to assess the association of SLE activity and thyroid diseases. A p-value less than 0.05 was considered significant.

Result The overall prevalence of thyroid dysfunctions was 26 (17.2\%) out of 151 SLE patients. The most common dysfunctions were subclinical hypothyroidism 11 (7.3\%) and hypothyroidism 7 (4.6\%). Hypothyroidism patients were found to have a positive and equally high frequency (57\%) of anti-Tg and anti-TPO, and equal frequency of a positive anti-Tg and anti-TPO (64\%) was found in subclinical hypothyroidism patients as well. No association was found between SLE activity and thyroid diseases.

Conclusion Our SLE patients had a high prevalence of subclinical hypothyroidism (7.3\%). No significant association between SLE activity and thyroid diseases.

Absatrct PS2:36 Table 1 Distribution of thyroid diseases and tabulation with thyroid antibodies

\begin{tabular}{l|c|c|c|c|c|c}
\hline \multirow{2}{*}{ Thyroid Status } & \multicolumn{2}{|c|}{$\begin{array}{c}\text { ANTI-TPO } \\
\text { n(n\%) }\end{array}$} & \multirow{2}{*}{ p-value } & \multicolumn{2}{c|}{$\begin{array}{c}\text { ANTI-Tg } \\
\text { n(n\%) }\end{array}$} & \multirow{2}{*}{ p-value } \\
\cline { 2 - 3 } & Positive & Negative & & Positive & Negative & \\
\hline Hyperthyroid & 0 & $1(100 \%)$ & & 0 & $1(100 \%)$ & \\
Hypothyroid & $4(57 \%)$ & $3(43 \%)$ & & $4(57 \%)$ & $3(43 \%)$ & \\
$\begin{array}{l}\text { Subclinical } \\
\text { Hyperthyroid }\end{array}$ & $3(60 \%)$ & $2(40 \%)$ & & $3(60 \%)$ & $2(40 \%)$ & \multirow{2}{*}{0.22} \\
$\begin{array}{l}\text { Subclinical } \\
\text { Hypothyroid }\end{array}$ & $7(64 \%)$ & $4(36 \%)$ & & $7(64 \%)$ & $4(36 \%)$ & \\
Sick Thyroid & 0 & $2(100 \%)$ & & 0 & $2(100 \%)$ & \\
Normal & $81(65 \%)$ & $44(35 \%)$ & & $83(66 \%)$ & $42(34 \%)$ & \\
\hline Total & $95(63 \%)$ & $56(37 \%)$ & & $96(64 \%)$ & $55(36 \%)$ & \\
\hline
\end{tabular}

Absatrct PS2:36 Table 2 Univariate analysis of the association of SLE activity and thyroid diseases

\begin{tabular}{l|l|c|c|c}
\hline & Thyroid Status & $\mathbf{n}$ & Mean \pm SD & p-value \\
\hline Anti-dsDNA & Diseased & 23 & $204.52 \pm 491.90$ & 0.775 \\
& Normal & 114 & $173.77 \pm 306.69$ & \\
C3 (0.9-1.8) & Diseased & 26 & $0.80 \pm 0.39$ & 0.729 \\
24hrs urine for & Normal & 124 & $0.77 \pm 0.59$ & \\
protien & Diseased & 21 & $1.96 \pm 3.77$ & 0.366 \\
Selena score & Normal & 118 & $1.18 \pm 2.05$ & \\
& Diseased & 26 & $15.62 \pm 10.06$ & 0.206
\end{tabular}

